\title{
Practical method for measurement and compensation of linear coupling driving term
}

\author{
M. Masaki, M. Takao, K. Soutome, and S. Takano \\ JASRI/SPring-8, 1-1-1 Kouto, Sayo, Hyogo, 679-5198, Japan
}

(Received 27 October 2008; published 19 February 2009)

\begin{abstract}
We demonstrated a method with practical value for the measurement and global compensation of a complex coupling driving term $C$ of linear difference resonance, using a turn-by-turn beam position monitor (BPM) at the SPring-8 storage ring. The method is based on the perturbation theory with the single-resonance approximation. The accurate complex $C$ was obtained from coefficients of the eigenmode expansion of the coupled betatron oscillation observed near a target linear difference resonance. The global compensation for the measured $C$ was successfully carried out by determining optimal strengths of the two independent correcting skew quadrupoles for generating a counterterm $-C$ without using empirical methods. Meanwhile, we then confirmed that the determined optimal strengths gave a minimum vertical beam size by scanning the strength of the correcting skew quadrupole field. To demonstrate the validity of our method, $C$ was also measured while varying the strengths of the correcting magnets around the optimal values to generate deliberate skew quadrupole error fields. We confirmed that the measured values of $C$ agreed with those coming from the deliberate error fields.
\end{abstract}

DOI: 10.1103/PhysRevSTAB.12.024002

PACS numbers: 29.27. $-\mathrm{a}, 41.85 .-\mathrm{p}$

\section{INTRODUCTION}

Betatron coupling is one of the essentials for machine performance in circular accelerators, such as electron storage rings for high-brilliance light sources and particle colliders of high luminosity. Strong coupling can induce optics distortion, shift of betatron tunes, and excitation of resonances. Accurate correction of the linear betatron coupling is of primary importance to improve the brilliance for a low-emittance light source. The correction also has a secondary effect that the excitation of other nonlinear resonances can be suppressed. For examples at the SPring-8 storage ring, the excitation of skew sextupole resonance $\left(2 \nu_{x}-\nu_{y}=62\right.$ near the user-time working point) was successfully suppressed by correcting the linear coupling resonance [1]. Furthermore, we also observed that the global correction of the linear coupling reduced impact on the vertical emittance of skew quadrupole error fields coming from undulators.

If a certain equipment installed in the ring causes the strong linear coupling, a local compensation of the skew quadrupole error field near the equipment can be effective. On the other hand, if the coupling comes from very weak skew error fields distributed along the large ring, such as the SPring-8 storage ring, the local compensation needs an elaborate correction technique. Recently, at the Paul Scherrer Institute Swiss Light Source, the precise error analysis was performed by using the measured response matrix, and an ultralow emittance coupling ratio of $0.05 \%$ has been successfully achieved by applying a singular value decomposition based local correction algorithm [2].

Skew quadrupole error fields can be generated by complicated factors such as alignment errors of normal quadrupole magnets and vertical offsets of a closed orbit distortion (COD) in normal sextupole magnets. Although the skew error fields are usually weak, the coupled betatron motions of a stored beam can be enhanced near a linear difference resonance line. Under such conditions, the perturbation theory with the single-resonance approximation is applicable, and the linear coupling can be corrected effectively by global compensation of the coupling driving term $C$.

Resonance driving terms were successfully measured by the fast-Fourier-transform (FFT) analysis of the turn-byturn beam position monitor (BPM) data at the CERN SPS and the BNL RHIC between 1999 and 2004 [3-6]. In particular, this technique is a powerful tool to measure the local sextupolar resonance driving terms [7]. The complex driving term $C$ of the linear coupling resonance has been also measured by the envelope analysis of coherent betatron oscillations excited by a horizontal kicker [8] or the skew quadrupole modulation technique [9], in addition to the FFT technique mentioned above. Also at the SPring8 storage ring, the complex $C$ was measured by using the coherent coupled betatron oscillations. We observed the coherent oscillations near a target linear difference resonance to improve the precision of the single-resonance approximation. The accurate driving term $C$ was extracted from both the horizontal and vertical turn-by-turn BPM signals by fitting procedure of the betatron motions expanded into two eigenmodes for the single linear coupling resonance.

For the global correction of the linear coupling, empirical methods of scanning two independent correcting skew fields have been used to achieve minimum betatron tune separation [10], minimum vertical beam size or maximum beat period of the coherent betatron oscillations, etc. However, these methods have no guarantee to correct the 
complex $C$ properly, because two free parameters have to be determined by one probe which gives us only the absolute value of $C$. Using both the amplitude and phase of the measured $C$, we can uniquely determine optimal strengths of the two independent correcting skew quadrupole magnets without the empirical means. Our simple correction technique for the linear coupling driving term has the advantage of being quick and precise, and has the practical significance for the machine tuning to improve beam quality. More precise correction including other distant coupling resonances which have only a smaller effect on the betatron motions will be realized by extending our scheme, but it is not mentioned in this paper.

In Sec. II, we briefly review the coupled betatron oscillation and the coupling driving term. The method of measuring the complex $C$ using a turn-by-turn BPM is described in Sec. III. In Sec. IV, we describe how to determine optimal strengths of the correcting skew quadrupole magnets using the measured value of $C$. A practical scheme for the global compensation of $C$ is also proposed, and the validity of our method is demonstrated.

\section{COUPLED BETATRON MOTION}

We assume that small skew quadrupole magnetic components are distributed as error fields along the circumference of a circular accelerator. In such a case, the horizontal $(x)$ and vertical $(y)$ betatron beam motions can be coupled. The strong coupled motion appears near the linear difference resonance $\nu_{x}-\nu_{y} \approx q$ (integer). If the skew quadrupole error fields are sufficiently small in comparison with the fields of main magnets composing a lattice of the ring, we can treat the coupled betatron motions with the Hamilton perturbation theory [10-13] under the singleresonance approximation.

According to the perturbation theory, the $x-y$ betatron beam motions are expressed by the following formulas [13]:

$$
x(s)=\left(A_{1} e^{-2 \pi i \nu_{1} s / L}+A_{2} e^{-2 \pi i \nu_{2} s / L}\right) \sqrt{\frac{\beta_{x}(s)}{2}} e^{i \phi_{x}(s)}+\text { c.c. },
$$

$$
\begin{aligned}
y(s)= & \frac{C}{2}\left(\frac{A_{1}}{\nu_{2}} e^{2 \pi i \nu_{2} s / L}+\frac{A_{2}}{\nu_{1}} e^{2 \pi i \nu_{1} s / L}\right) \sqrt{\frac{\beta_{y}(s)}{2}} e^{i \phi_{y}(s)} \\
& + \text { c.c. },
\end{aligned}
$$

where c.c. denotes the complex conjugate of the preceding term, and $\beta_{x, y}(s), \phi_{x, y}(s), A_{1,2}, L$, and $s$ are the betatron functions, phase advances, integration constants, circumference of the ring, and beam path length, respectively. The shifts $\nu_{1,2}$ of unperturbed betatron tunes $\nu_{x, y}$ are written as

$$
\nu_{1,2}=\frac{1}{2}\left(\Delta \pm \sqrt{\Delta^{2}+|C|^{2}}\right),
$$

where $\Delta=\nu_{x}-\nu_{y}-q$. The complex number $C$ in Eq. (2) is referred to as the coupling driving term and is denoted by the following formula:

$$
\begin{aligned}
C & =\frac{1}{2 \pi} \int_{0}^{L} K_{\mathrm{err}}(s) \sqrt{\beta_{x}(s) \beta_{y}(s)} e^{i\left[\phi_{x}(s)-\phi_{y}(s)-(2 \pi s / L) \Delta\right]} d s \\
& \equiv|C| e^{i \phi_{c}},
\end{aligned}
$$

where $K_{\text {err }}(s)$ is the field strength of the distributed skew quadrupole errors, and the $\Delta$ dependence of $C$ is small near the linear difference resonance where $\Delta$ is close to zero and $2 \pi \Delta \cdot s / L \ll \phi_{x}-\phi_{y}$. The absolute value and phase of $C$ dominate the amplitude and phase of the coupled vertical oscillation, respectively, induced from the horizontal oscillation.

\section{DERIVATION OF COUPLING DRIVING TERM C FROM TURN-BY-TURN BPM DATA}

We measured the coupling driving term $C$ of the SPring8 storage ring using a turn-by-turn BPM system [14]. The coherent betatron oscillations expressed by Eqs. (1) and (2) were induced by pinging a single-bunch beam stored in the storage ring in the horizontal direction with a pulsed bump magnet for beam injection. The betatron oscillations were then observed using the turn-by-turn BPMs located at about every two cells of the storage ring. To increase sensitivity in measuring the vertical oscillation induced by the betatron coupling, we chose a working point near the difference resonance line, where the single-resonance approximation is sufficiently applicable.

Equations (1) and (2) are reduced to the following forms, suitable to fit the measured turn-by-turn BPM data:

$$
\begin{aligned}
x_{n}= & e^{-n D}\left\{A_{u} \cos \left(2 \pi n \nu_{u}+\Phi_{u}\right)\right. \\
& \left.+A_{v} \cos \left(2 \pi n \nu_{v}+\Phi_{v}\right)\right\},
\end{aligned}
$$

$y_{n}=e^{-n D}\left\{B_{u} \cos \left(2 \pi n \nu_{u}+\Psi_{u}\right)+B_{v} \cos \left(2 \pi n \nu_{v}+\Psi_{v}\right)\right\}$,

where the exponential damping factor $e^{-n D}$ comes from the nonlinear filamentation of coherent betatron oscillations with a Lorentz tune distribution [15], $\nu_{u}$ and $\nu_{v}$ are tunes of the eigenmodes $(u, v)$, and $n$ is the turn number. Parameters $A_{u}, A_{v}, B_{u}, B_{v}$ and $\Phi_{u}, \Phi_{v}, \Psi_{u}, \Psi_{v}$ express amplitudes and phases of the oscillations, respectively. Note that the horizontal and vertical betatron oscillations are given by the linear combination of two eigenmodes $(u, v)$. Free parameters in the fitting process are $A_{u}, A_{v}, B_{u}$, $B_{v}, \Phi_{u}, \Phi_{v}, \Psi_{u} \Psi_{v}, \nu_{u}, \nu_{v}$, and $D$. Figure 1 shows an example of the turn-by-turn BPM data with the fitted curves. We can observe an amplitude modulation with frequency $\left|\nu_{u}-\nu_{v}\right| / T_{\text {rev }}$ in the $x$ and $y$ directions, where $T_{\text {rev }}$ is the revolution period. The modulation in the $x$ and $y$ directions has a phase difference of $90^{\circ}$. This is typical of a 

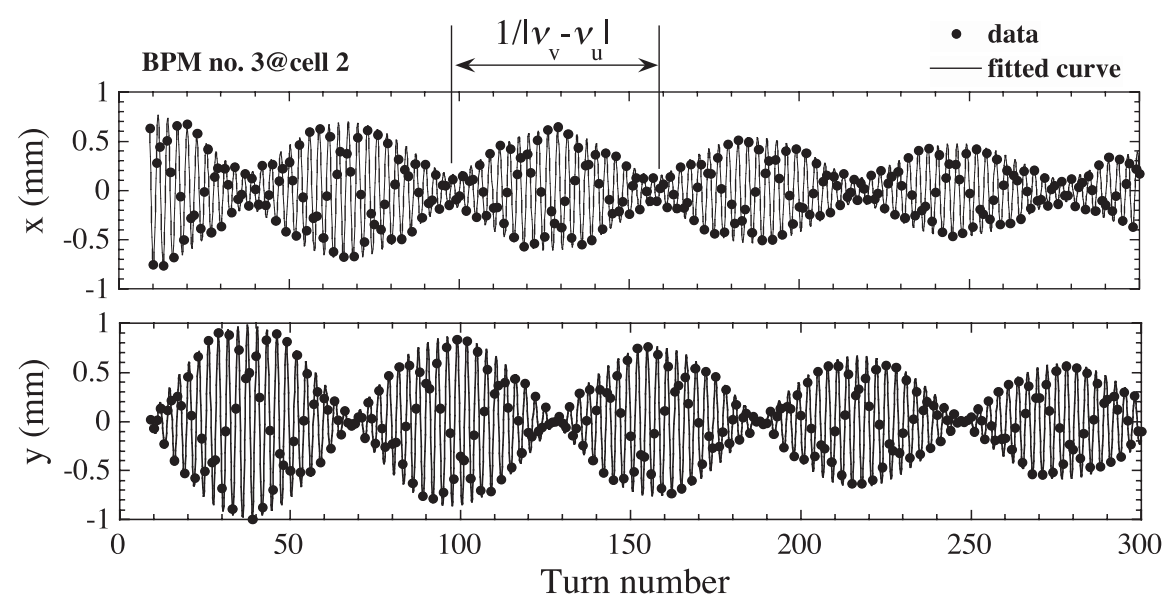

FIG. 1. An example of the turn-by-turn BPM data (dots) measured at the working point $\left(\nu_{x}, \nu_{y}\right)=(40.3509,18.3477)$ near the linear difference resonance. The solid lines show the curves of Eqs. (5) and (6) fitted to the data.

coupling resonance where energies of the $x-y$ betatron oscillations alternate between two directions.

As other data analysis instead of the fitting procedure, the FFT analysis can be also employed to obtain the equivalent of the parameters $A_{u}, A_{v}, B_{u}, B_{v}, \Phi_{u}, \Phi_{v}$, $\Psi_{u}, \Psi_{v}, \nu_{u}, \nu_{v}$ mentioned above. In Fig. 2, the FFT analyses of the horizontal and vertical turn-by-turn data in Fig. 1 are shown as a typical example. In the amplitude spectrum, we can see only two peaks corresponding to the eigenmodes $(u, v)$. This supports validity of the model functions [Eqs. (5) and (6)] with the single-resonance approximation. The phase spectrum has steep slopes around the fractional tunes at the two peaks of the amplitude spectrum. We note that the accuracy of an extracted phase can be limited by this steeply inclined behavior, since the width of a frequency bin is dominated by the number of truncated data points from the turn-by-turn BPM. Although the frequency resolution can be improved by extending the original data by zeros, the proper zeroregion size will need to be estimated. We chose the straightforward fitting analysis which does not need the artificial means like the zero padding to solve the issue of the frequency resolution.

The absolute value $|C|$ was derived from the fitted eigentunes $\nu_{u}, \nu_{v}$ and amplitude parameters $A_{u}, A_{v}, B_{u}$, and $B_{v}$. To show this, we first note that the amplitude parameters can be expressed as

$$
\begin{aligned}
A_{u}=\sqrt{2 \beta_{x}}\left|A_{2}\right|, & A_{v}=\sqrt{2 \beta_{x}}\left|A_{1}\right|, \\
B_{u}=|C| \sqrt{\frac{\beta_{y}}{2}} \frac{\left|A_{2}\right|}{\nu_{1}}, & B_{v}=|C| \sqrt{\frac{\beta_{y}}{2}} \frac{\left|A_{1}\right|}{\nu_{2}} .
\end{aligned}
$$

We then define $\zeta$ as

$$
\zeta \equiv \frac{A_{u} B_{v}}{A_{v} B_{u}}=\frac{\nu_{1}}{\nu_{2}}=\frac{\Delta+\sqrt{\Delta^{2}+|C|^{2}}}{\Delta-\sqrt{\Delta^{2}+|C|^{2}}} .
$$

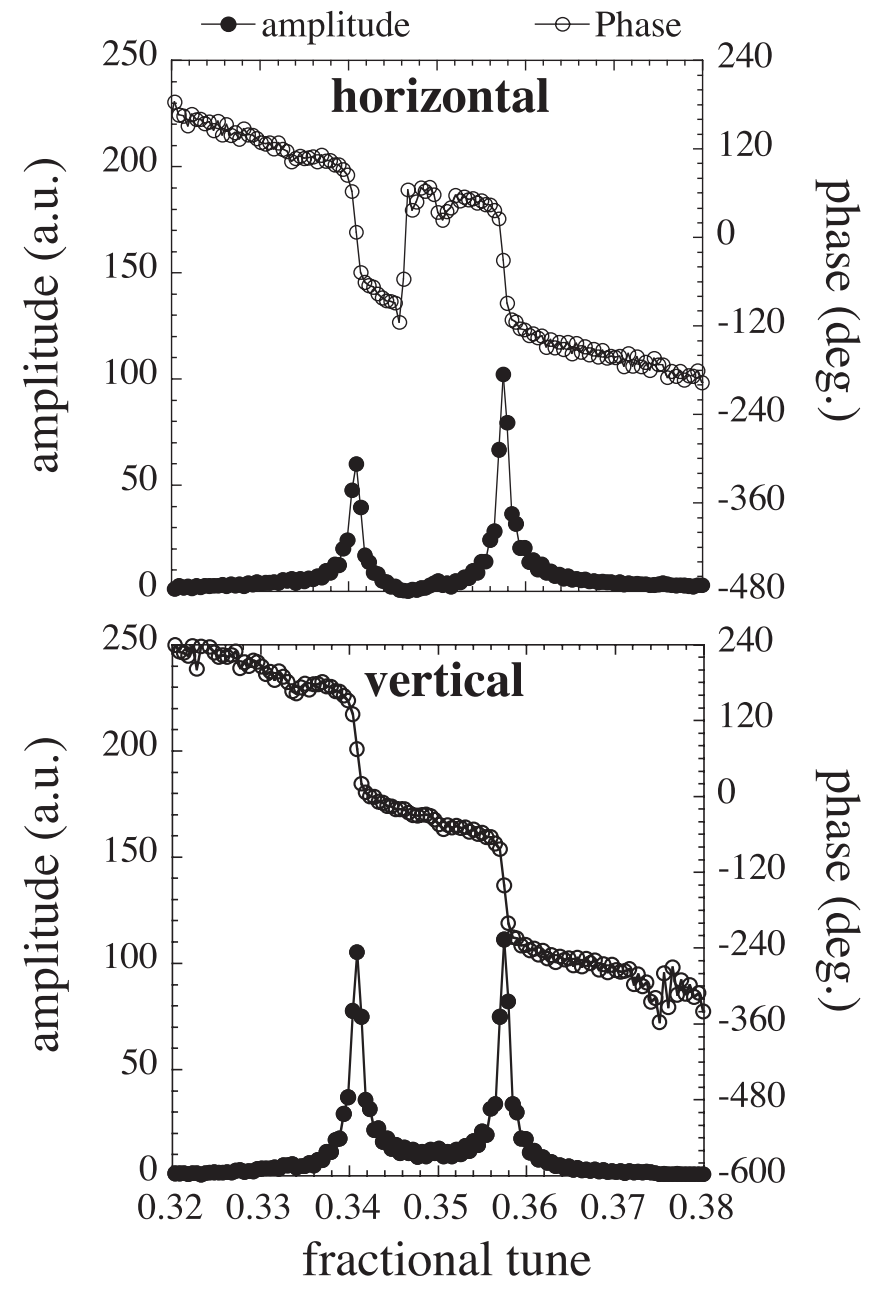

FIG. 2. FFT analyses of the horizontal and vertical turn-byturn BPM data shown in Fig. 1. The turn-by-turn data are truncated at 1024 turns. Filled and open circles with solid lines show the amplitude and phase spectra, respectively. We see that the amplitude spectrum has the two peaks corresponding to the eigenmodes $(u, v)$ and the phase spectrum has the steeply inclined behaviors around the fractional tunes at the two peaks. 


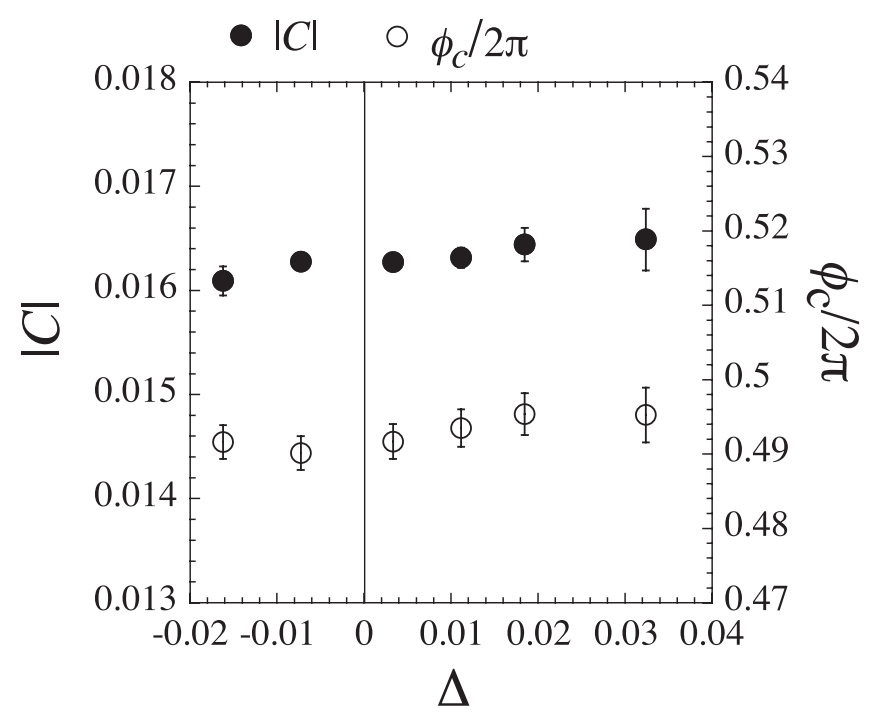

FIG. 3. The absolute values (filled circles) and phases (open circles) of $C$ measured at several working points near the linear difference resonance. We see that the dependence of the measured $C$ on the working points can be neglected.

The eigentunes $\nu_{u}, \nu_{v}$ for $\Delta>0$ can be written as

$$
\nu_{u}=\nu_{x}-\nu_{2}, \quad \nu_{v}=\nu_{y}+\nu_{2}
$$

and those for $\Delta<0$ as

$$
\nu_{u}=\nu_{x}-\nu_{1}, \quad \nu_{v}=\nu_{y}+\nu_{1}
$$

From Eqs. (10) and (11), we obtain

$$
\Delta=-\left|\nu_{u}-\nu_{v}\right| /\left(\frac{1-\zeta}{1+\zeta}\right)
$$

From Eqs. (9) and (12), we obtain the expression for $|C|$ :

$$
|C|=\left|\nu_{u}-\nu_{v}\right| \sqrt{1-\left(\frac{1+\zeta}{1-\zeta}\right)^{2}}
$$

The phase $\phi_{c}$ of $C$ was derived from the fitted phase parameters $\Phi_{u}, \Phi_{v}, \Psi_{u}$, and $\Psi_{v}$ which can be expressed as

$$
\begin{aligned}
& \Phi_{u}=\phi_{x}+\phi_{1}-2 \pi \nu_{1} \frac{s_{i}}{L}, \\
& \Phi_{v}=\phi_{x}+\phi_{2}-2 \pi \nu_{2} \frac{s_{i}}{L},
\end{aligned}
$$

$$
\begin{aligned}
& \Psi_{u}=\phi_{c}+\phi_{y}+\phi_{1}+2 \pi \nu_{2} \frac{s_{i}}{L} \\
& \Psi_{v}=\phi_{c}+\phi_{y}+\phi_{2}+2 \pi \nu_{1} \frac{s_{i}}{L}
\end{aligned}
$$

where $\phi_{1}$ and $\phi_{2}$ show the phases of the complex numbers $A_{1}$ and $A_{2}$, respectively, and $s_{i}$ denotes the beam path length at the $i$ th BPM. From Eqs. (14) and (15), we obtain

$$
\phi_{c}=\left\{\begin{array}{l}
\Psi_{u}-\Phi_{u}+\phi_{x}-\phi_{y}-2 \pi \Delta \cdot s_{i} / L \\
\text { or } \\
\Psi_{v}-\Phi_{v}+\phi_{x}-\phi_{y}-2 \pi \Delta \cdot s_{i} / L
\end{array}\right.
$$

where we used design values for $\phi_{x}$ and $\phi_{y}$ of the unperturbed system.

The fitting was carried out for the data from each turnby-turn BPM, so we obtained as many experimentally derived $C$ values as the number of the BPMs. Since $C$ is independent of the path length $s_{i}$, we adopted the average of the complex values $C=|C| e^{i \phi_{c}}$ from all the BPMs as a measured value of the coupling driving term. Figure 3 shows $|C|$ and $\phi_{c}$ measured at several working points near the linear difference resonance line. As expected, the $\Delta$ dependence of $C$ is small near the resonance line, and we can see in Fig. 3 that the measured $C$ does not depend on the working points. By averaging the complex $C$ measured at all the working points, we obtained the real part $\operatorname{Re}(C)=-0.01630 \pm 0.00006$ and the imaginary part $\operatorname{Im}(C)=0.00072 \pm 0.00011$, and consequently $|C|=0.01631 \pm 0.00006$ and $\phi_{c}=(0.4930 \pm$ $0.0011) \times 2 \pi$.

\section{DETERMINATION OF FIELD STRENGTH OF CORRECTING SKEW QUADRUPOLE MAGNETS}

The SPring-8 storage ring is composed of 36 normal Chasman-Green cells (CGCs) and four magnet-free long straight sections (LSSs) of $27 \mathrm{~m}$ with matching sections [16]. Two-family skew quadrupole magnets for the global coupling correction are installed at the achromatic arcs (ARCs) of the CGCs every two cells and at both ends of all the LSSs. A compensation term $C_{\mathrm{SK}}$ for the coupling driving term $C$ is obtained by replacing $K_{\text {err }}$ of Eq. (4) with the strengths of the correcting skew quadrupole magnets of the ARC and the LSS, and is denoted by the following formula:

$$
C_{\mathrm{SK}}=\frac{1}{2 \pi}\left\{\frac{K_{\mathrm{ARC}}}{N_{\mathrm{ARC}}} \sum_{j=\mathrm{ARC}} \sqrt{\beta_{x}\left(s_{j}\right) \beta_{y}\left(s_{j}\right)} e^{i\left[\phi_{x}\left(s_{j}\right)-\phi_{y}\left(s_{j}\right)-\left(2 \pi s_{j} / L\right) \Delta\right]}+\frac{K_{\mathrm{LSS}}}{N_{\mathrm{LSS}}} \sum_{j=\mathrm{LSS}} \sqrt{\beta_{x}\left(s_{j}\right) \beta_{y}\left(s_{j}\right)} e^{i\left[\phi_{x}\left(s_{j}\right)-\phi_{y}\left(s_{j}\right)-\left(2 \pi s_{j} / L\right) \Delta\right]}\right\},
$$

where $K_{\mathrm{ARC}}$ and $K_{\mathrm{LSS}}$ are the total integrated strengths of the correcting skew quadrupole magnets of the ARC and LSS, respectively, $N_{\mathrm{ARC}}$ and $N_{\mathrm{LSS}}$ are the total number of the skew magnets of the ARC and LSS, respectively. At the
SPring-8 storage ring, $N_{\mathrm{ARC}}=20$ and $N_{\mathrm{LSS}}=8$. The phase $\phi_{x}-\phi_{y}$ in Eq. (17) approximately has a difference of $m \pi$ between the skew magnets in the adjacent two ARCs or two LSSs, and a difference of $(m+1 / 2) \pi$ be- 
tween the skew magnet in the ARC and that in the LSS, where $m$ is an integer. These phase differences indicate that the first and second compensation terms of Eq. (17) are in quadrature. Requiring $C_{\mathrm{SK}}$ to be equal to $-C$, we can uniquely determine the total integrated strengths $K_{\mathrm{ARC}}$ and $K_{\mathrm{LSS}}$ by solving a linear equation (17). We used design values for the betatron functions $\beta_{x, y}$, the phase advances $\phi_{x, y}$, and $\Delta$ in the unperturbed system. The distortion of betatron functions has been corrected with auxiliary power supplies to normal quadrupole magnets and found to be sufficiently small, being about $2 \%$ by the response matrix analysis [17]. As a result, we obtained $K_{\mathrm{ARC}}=$ $-0.00163 \pm 0.00004\left(\mathrm{~m}^{-1}\right)$ and $K_{\mathrm{LSS}}=0.00338 \pm$

$\sigma_{\mathrm{y}}$ by XBI

$\times \mathrm{ICl}$ by turn-by-turn BPM

- $\sigma_{\mathrm{y}}$ by $2 \mathrm{D}$-interferometer
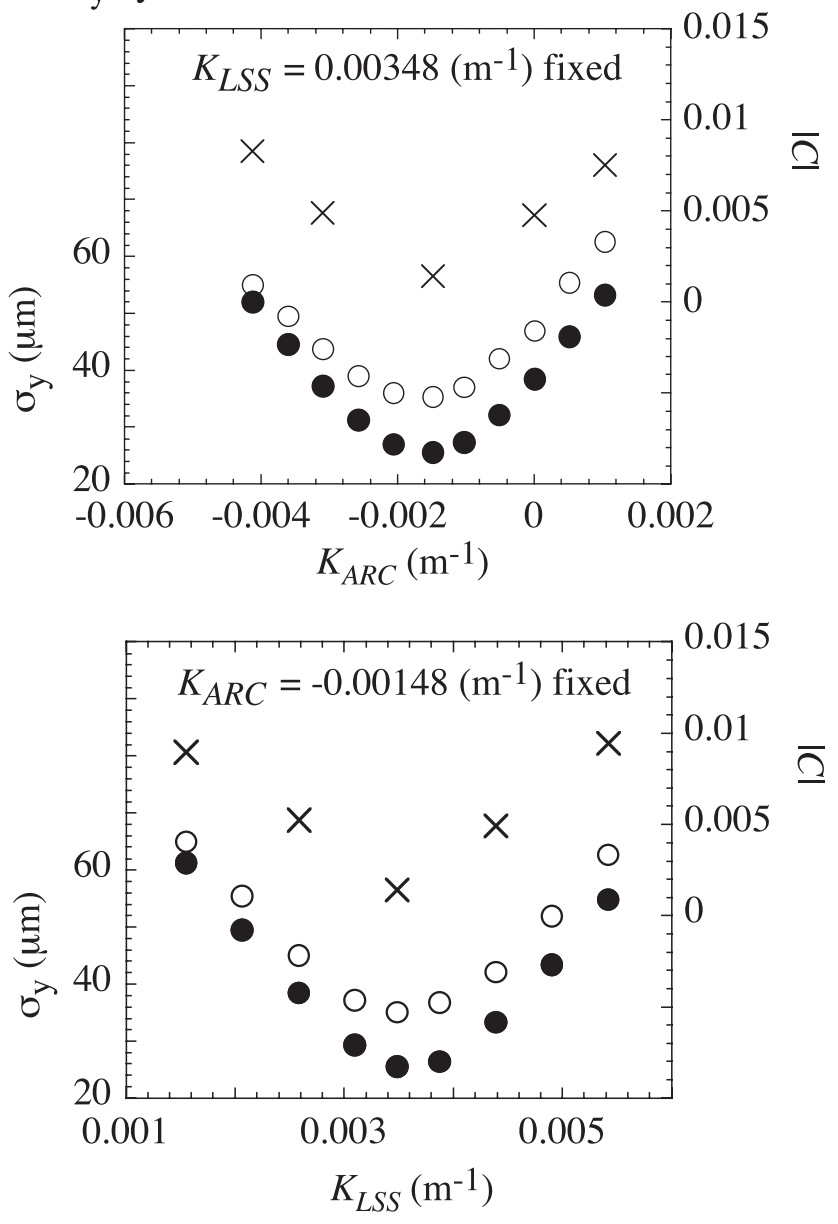

FIG. 4. Vertical beam sizes measured by the XBI (open circles) and the 2D interferometer (filled circles), and $|C|$ (crosses) measured by the turn-by-turn BPM as a function of $K_{\mathrm{ARC}}$ and $K_{\mathrm{LSS}}$. For both beam-size monitors of XBI and 2D interferometer, the minimum vertical beam sizes are given when $\left(K_{\mathrm{ARC}}, K_{\mathrm{LSS}}\right)$ are $\left(-0.00148 \mathrm{~m}^{-1}, 0.00348 \mathrm{~m}^{-1}\right)$, where the measured $|C|$ also becomes minimum. The working point was chosen at $\left(\nu_{x}, \nu_{y}\right)=(40.3818,18.3451)$, near the difference resonance. $0.00001\left(\mathrm{~m}^{-1}\right)$ using the measured value of $C$ described in Sec. III.

To check our results, we scanned the strengths $K_{\mathrm{ARC}}$ and $K_{\mathrm{LSS}}$ to minimize the vertical beam size $\sigma_{y}$. If the betatron coupling makes a dominant contribution to $\sigma_{y}$, it is expressed by the following formula $[10,13]$ :

$$
\sigma_{y}=\sqrt{\frac{\frac{1}{2}|C|^{2}}{\Delta^{2}+|C|^{2}} \beta_{y} \epsilon_{0}},
$$

where $\epsilon_{0}$ is a natural emittance in the unperturbed system. Equation (18) indicates that $|C|$ also becomes minimum when the minimum vertical beam size is realized. The vertical beam sizes were measured by the $\mathrm{x}$-ray beam imager (XBI) [18] and the two-dimensional synchrotron radiation interferometer (2D interferometer) [19] using bending magnet radiation. These are shown in Fig. 4 as a function of $K_{\mathrm{ARC}}$ and $K_{\mathrm{LSS}}$ together with the absolute values of $C$ measured by the turn-by-turn BPM. We chose a working point near the difference resonance to increase the response sensitivity of the vertical beam size to the skew fields. The skew field strengths $K_{\mathrm{ARC}}$ and $K_{\mathrm{LSS}}$ giving the minimum vertical beam size were $-0.00148\left(\mathrm{~m}^{-1}\right)$ and $0.00348\left(\mathrm{~m}^{-1}\right)$, respectively, where the measured $|C|$ also became minimum. The optimum strengths of the correcting skew magnets determined from the complex $C$ are consistent with those giving the minimum vertical beam size, but we can see a slight discrepancy between the two results. Possible explanations of the discrepancy are as follows: as the dominant linear coupling driving term is compensated due to the correcting skew quadrupole magnets, (i) effects of other distant coupling resonance or higher-order resonance terms on $\sigma_{y}$ grow relatively and Eq. (17) is no longer applicable for the compensation of such resonance terms, or (ii) effect of the correcting skew quadrupole fields on the vertical dispersion contributing to $\sigma_{y}$ becomes not negligible, and consequently Eq. (18) becomes inapplicable. The small discrepancy, however, will not be essential for a practical coupling correction scheme that we propose: the first step of the correction scheme is to measure the complex coupling driving term $C$ using the turn-by-turn BPM; the second step is to set the correcting skew field strengths $K_{\mathrm{ARC}}$ and $K_{\mathrm{LSS}}$ to give the counterterm $-C$; and the third step is to carry out the first and second steps iteratively until the measured $C$ converges within the error of measurement.

Finally, to demonstrate the validity of our method, we measured the complex $C$ while the skew quadrupole field strengths $\left(K_{\mathrm{ARC}}, K_{\mathrm{LSS}}\right)$ were deliberately varied around the optimum values. Figure 5 shows the comparison between the experimental value $C_{\exp }$ measured using the turn-byturn BPM and the calculated value $C_{\text {cal }}=C_{\mathrm{SK}}+C_{0}$ obtained from Eq. (17) with the given skew quadrupole field strengths. The offset $C_{0}$ is the measured value of $C$ at $K_{\mathrm{ARC}}=0$ and $K_{\mathrm{LSS}}=0$ shown in Sec. III. We can see that $C_{\exp }$ agrees well with $C_{\text {cal }}$. 


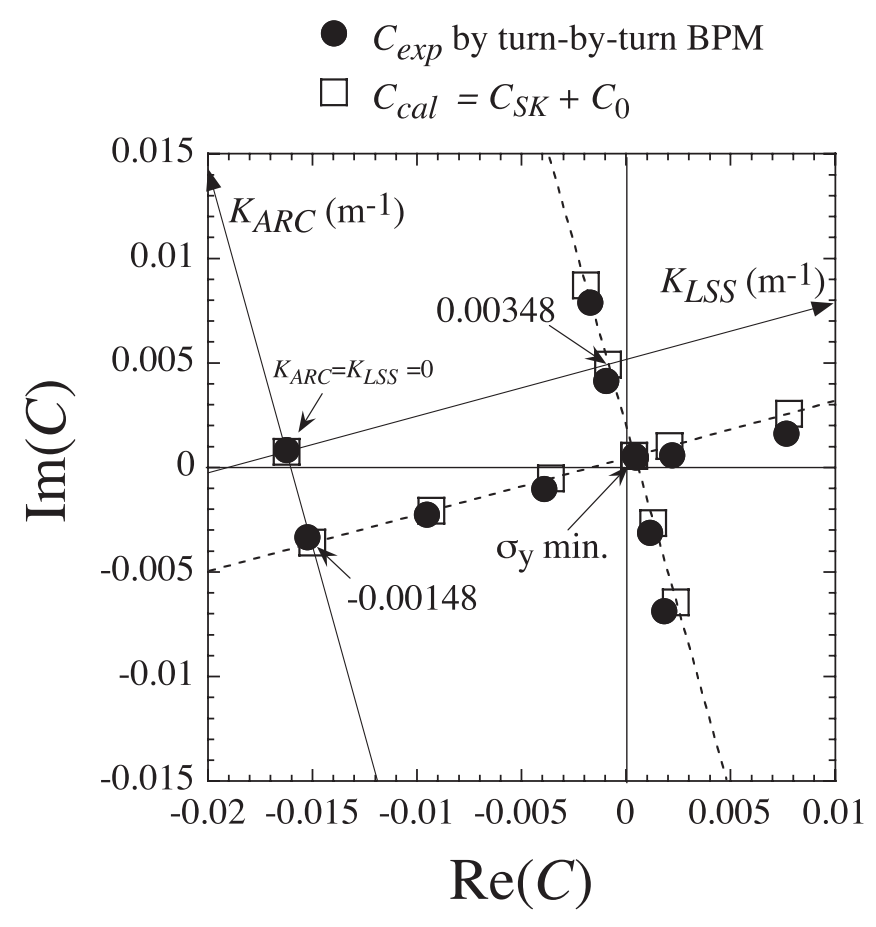

FIG. 5. The measured values $C_{\text {exp }}$ (filled circles) as a function of $\left(K_{\mathrm{ARC}}, K_{\mathrm{LSS}}\right)$ are compared with $C_{\mathrm{cal}}$ (open squares) calculated from the given skew field strength. The working point was $\left(\nu_{x}, \nu_{y}\right)=(40.3509,18.3477)$. The measured values are almost consistent with the calculations. The minimum absolute value of $C$ was given at $\left(K_{\mathrm{ARC}}, K_{\mathrm{LSS}}\right)=\left(-0.00148 \mathrm{~m}^{-1}, 0.00348 \mathrm{~m}^{-1}\right)$, where the minimum vertical beam size was realized, as shown in Fig. 4.

\section{SUMMARY}

The complex driving term $C$ for the linear coupling was measured using the turn-by-turn BPM system at the SPring- 8 storage ring. The optimal strengths of the twofamily correcting skew magnets of the ARC and LSS were directly determined for generating the counterterm $-C$ without using the empirical means. The determined strengths were consistent with those obtained by minimizing the vertical beam size. We also proposed a practical correction scheme of global coupling using the measured $C$. The validity of our measuring and correcting method was experimentally confirmed by comparing the measured $C$ as a function of the strengths of the correcting skew quadrupole magnets with the calculated values. Since our technique allows quick-and-precise measurement and compensation of the driving term $C$, it will become a practical tool for the proper feedback to the excitation parameters of the two-family skew quadrupole magnets in the global coupling correction.

\section{ACKNOWLEDGMENTS}

The authors would like to thank Dr. C. Zhang and Dr. K. Tsumaki for setting up the skew quadrupole magnets of the four long straight sections, which are indispensable for the global coupling correction.

[1] M. Takao, M. Masaki, J. Schimizu, K. Soutome, and S. Takano, in Proceedings of the 2007 Particle Accelerator Conference, Albuquerque, New Mexico, 2007 (IEEE, Albuquerque, New Mexico, 2007), p. 4003.

[2] A. Andersson, M. Böge, A. Lüdeke, and A. Streun, in Proceedings of the 11th European Particle Accelerator Conference, Genoa, 2008 (EPS-AG, Genoa, Italy, 2008), p. 1983.

[3] R. Bartolini, L. H. A. Leunissen, Y. Papaphilippou, F. Schmidt, and A. Verdier, in Proceedings of the 18th Particle Accelerator Conference, New York, 1999 (IEEE, New York, 1999), p. 1557.

[4] F. Schmidt, R. Tomas, and A. Faus-Golfe, in Proceedings of the Particle Accelerator Conference, Chicago, IL, 2001 (IEEE, New York, 2001), p. 437.

[5] M. Hayes, F. Schmidt, and R. Tomas, in Proceedings of the 8th European Particle Accelerator Conference, Paris, 2002 (EPS-IGA and CERN, Geneva, 2002), p. 1290.

[6] R. Tomas, M. Bai, R. Calaga, and W. Fischer, Phys. Rev. ST Accel. Beams 8, 024001 (2005).

[7] F. Schmidt, M. Hayes, and R. Tomas, in Proceedings of the 20th Particle Accelerator Conference, Portland, OR, 2003 (IEEE, New York, 2003), p. 2231.

[8] S. Y. Lee, Accelerator Physics (World Scientific, Singapore, 2004), 2nd ed., p. 193.

[9] Y. Luo, P. Cameron, A. Della Penna, Jr., J. Laster, R. Lee, A. Marusic, F. Pilat, T. Roser, D. Trbojevic, and J. Wei, Phys. Rev. ST Accel. Beams 8, 014001 (2005).

[10] G. Guignard, Phys. Rev. E 51, 6104 (1995).

[11] A. Schoch, CERN Report No. 57-21, 1958.

[12] F. Willeke and G. Ripken, in Physics of Particle Accelerators, AIP Conf. Proc. No. 184 (AIP, New York, 1989), p. 758.

[13] M. Takao, Phys. Rev. ST Accel. Beams 9, 084002 (2006).

[14] S. Sasaki, SPring-8 Annual Report, p. 32 (1994).

[15] A. Hofmann, CERN Report No. 95-06, p. 283, 1995.

[16] H. Tanaka, K. Soutome, M. Takao, M. Masaki, H. Ohkuma, N. Kumagai, and J. Schimizu, Nucl. Instrum. Methods Phys. Res., Sect. A 486, 521 (2002).

[17] G. Liu, K. Kumagai, N. Kumagai, H. Ohkuma, K. Soutome, M. Takao, and H. Tanaka, Proceedings of the 18th Particle Accelerator Conference, New York, 1999 (Ref. [3]), p. 2337.

[18] S. Takano, M. Masaki, and H. Ohkuma, Nucl. Instrum. Methods Phys. Res., Sect. A 556, 357 (2006).

[19] M. Masaki and S. Takano, J. Synchrotron Radiat. 10, 295 (2003). 